\title{
Laser-ionized, beam-driven, underdense, passive thin plasma lens
}

\author{
C. E. Doss $\odot,{ }^{1, *}$ E. Adli, ${ }^{2}$ R. Ariniello $\odot,{ }^{1}$ J. Cary $\odot,{ }^{1,3}$ S. Corde $\odot,{ }^{4}$ B. Hidding,${ }^{5,6}$ M. J. Hogan $\odot,{ }^{7}$ \\ K. Hunt-Stone, ${ }^{1}$ C. Joshi, ${ }^{8}$ K. A. Marsh, ${ }^{8}$ J. B. Rosenzweig, ${ }^{9}$ \\ N. Vafaei-Najafabadi, ${ }^{10}$ V. Yakimenko, ${ }^{7}$ and M. Litos ${ }^{1}$ \\ ${ }^{1}$ University of Colorado Boulder, Department of Physics, Center for Integrated Plasma Studies, \\ Boulder, Colorado 80309, USA \\ ${ }^{2}$ University of Oslo, Department of Physics, 0316 Oslo, Norway \\ ${ }^{3}$ Tech-X Corporation, Boulder, Colorado 80301, USA \\ ${ }^{4}$ LOA, ENSTA Paris, CNRS, Ecole Polytechnique, \\ Institut Polytechnique de Paris, 91762 Palaiseau, France \\ ${ }^{5}$ Scottish Universities Physics Alliance, Department of Physics, University of Strathclyde, \\ Glasgow G4 ONG, United Kingdom \\ ${ }^{6}$ Cockcroft Institute, Sci-Tech Daresbury, Keckwick Lane, Daresbury, \\ Cheshire WA4 4AD, United Kingdom \\ ${ }^{7}$ SLAC National Accelerator Laboratory, Menlo Park, California 94025, USA \\ ${ }^{8}$ University of California Los Angeles, Department of Electrical Engineering, \\ Los Angeles, California 90095, USA \\ ${ }^{9}$ University of California Los Angeles, Department of Physics and Astronomy, \\ Los Angeles, California 90095, USA \\ ${ }^{10}$ Stony Brook University, Department of Physics and Astronomy, Stony Brook, New York 11794, USA
}

(Received 20 August 2019; published 7 November 2019)

\begin{abstract}
We present a laser-ionized, beam-driven, passive thin plasma lens that operates in the nonlinear blowout regime. This thin plasma lens provides axisymmetric focusing for relativistic electron beams at strengths unobtainable by magnetic devices. It is tunable, compact, and it imparts little to no spherical aberrations. The combination of these features make it more attractive than other types of plasma lenses for highly divergent beams. A case study is built on beam matching into a plasma wakefield accelerator at SLAC National Accelerator Laboratory's FACET-II facility. Detailed simulations show that a thin plasma lens formed by laser ionization of a gas jet reduces the electron beam's waist beta function to half of the minimum value achievable by the FACET-II final focus magnets alone.
\end{abstract}

DOI: 10.1103/PhysRevAccelBeams.22.111001

\section{INTRODUCTION}

Plasma-based accelerators offer accelerating gradients orders of magnitude greater than conventional metallic radio-frequency accelerating structures. In a plasma wakefield accelerator (PWFA), an electron drive beam generates a plasma wake as it propagates through a plasma, and a witness electron beam, located in the rear of the wake, is accelerated by the strong longitudinal wakefield [1-4]. When operating in the highly nonlinear blowout regime, an ion cavity is formed behind the head of the drive beam that is completely void of electrons. The ions in the cavity maintain an approximately uniform density

\footnotetext{
*Christopher.Doss@Colorado.edu
}

Published by the American Physical Society under the terms of the Creative Commons Attribution 4.0 International license. Further distribution of this work must maintain attribution to the author(s) and the published article's title, journal citation, and DOI. that is equal to the neutral plasma density. Inside the wake, the beam electrons experience an axisymmetric, linear transverse focusing force due to the Coulomb potential of the ions, leading to betatron oscillation of the beams' envelopes [1,5]. The betatron oscillation frequency depends on the energy of the particles, and therefore a beam with finite energy spread will experience chromatic phase spreading and projected emittance growth over many betatron periods in the plasma. To avoid this chromatic emittance growth, a beam must be matched to the plasma such that its divergence is exactly balanced against the focusing force of the ion cavity and no oscillations of the envelope occur [6-9].

The matching condition for a given plasma source depends on the density of the plasma and the energy of the beam. The condition is met when $\beta=k_{\beta}^{-1}$, where $\beta$ is the betatron Courant-Snyder parameter, $k_{\beta}=k_{p} / \sqrt{2 \gamma_{b}}$ is the betatron wave number inside the plasma, $k_{p}$ is the plasma wave number, and $\gamma_{b}$ is the relativistic Lorentz 
factor of the electron beam. The plasma wave number is given by $k_{p}^{2}=2 \pi r_{e} n_{p}$ in cgs units, where $r_{e}$ is the classical electron radius, and $n_{p}$ is the plasma number density. Most PWFA plasma sources have a density in the range $10^{16} \mathrm{~cm}^{-3}<n_{p}<10^{17} \mathrm{~cm}^{-3}$. At these densities the matched beta function is on the order of millimeters to a few centimeters for ultrarelativistic beams, which can be challenging to achieve in practice.

Conventional electromagnetic quadrupole focusing systems struggle to achieve such small beta functions due to transverse magnetic gradients that are generally limited to $\sim 1 \mathrm{~T} / \mathrm{m}$ and magnet lengths on the order of a meter. Permanent magnet quadrupoles (PMQs) can provide transverse gradients on the order of $500 \mathrm{~T} / \mathrm{m}$ [10], though they are limited to centimeters in length. These magnets typically suffer the drawbacks of smaller apertures and fixed magnet strength; however, progress has been made in developing PMQs with variable focusing strength [11]. Another focusing scheme that is often discussed in the literature is the use of gradual plasma density ramps at the entrance and exit of the PWFA plasma source [8,9,12-17]. This method should work in principle, but precise control over the plasma density profile is challenging, and beam matching has yet to be demonstrated using this technique. In this work, we show that the underdense, passive thin plasma lens can readily provide the necessary focusing for beam matching into a PWFA. At a plasma density of $10^{17} \mathrm{~cm}^{-3}$ (typical PWFA density), a relativistic electron beam will experience a focusing force in the plasma lens equivalent to a gradient of $3 \mathrm{MT} / \mathrm{m}$ in a quadrupole magnetic, which cannot be achieved by magnetic devices.

Plasma lenses have been considered and used in a number of different regimes and applications over the past few decades. One early conceptual application was for the final focus of a high-energy particle collider [18]. Plasma lenses have been primarily studied in the context of the overdense regime [19-21], wherein the plasma density exceeds the electron beam density and the resultant plasma response is linear or quasilinear, resulting in a nonlinear focusing response. Active plasma lenses [22-24] operate in this regime and utilize an applied, axial current in a discharge breakdown plasma source. The axial current produces a focusing azimuthal magnetic field that is adjusted through the operating current and plasma density of the device. These lenses have the drawback of limited transverse density uniformity leading to spherical aberrations as well as a tendency to transition to the nonlinear regime when used with intense electron beams [25].

Here, we consider the case of thin, passive plasma lenses operating in the underdense regime [12]. Previous experimental use of underdense lenses includes a beam interacting with a plasma jet outflow [26] and the laser-driven plasma lens at the exit of a laser-driven plasma wakefield accelerator [27,28]. The aforementioned experiments share some semblance with the scheme proposed here, with a few key differences: the thin plasma lens proposed in this work is intended to be operated in the highly nonlinear blowout regime, it is preionized by an external laser pulse, and is driven by an electron beam. This specific combination of characteristics permits several advantages over other electron beam focusing schemes. It is analytically modeled with ease; chromatic phase mixing in the plasma is negligible; focusing is linear across the full extent of the beam; it provides the strongest possible focusing per unit length and per individual element; it is extremely compact; it is easily tunable; and as with most plasma lenses, the focusing is axisymmetric. In this work, we describe an experimental setup for such a plasma lens and present simulation results that demonstrate its performance capabilities in a realistic experimental context modeled on the upcoming Facility for Advanced Experimental Accelerator Tests II (FACET-II) at SLAC National Accelerator Laboratory.

\section{ANALYTIC MODEL}

In the model presented here, the electron beam is assumed to have a two-bunch structure appropriate for use in a PWFA. This includes a leading drive beam that will drive the wake in both the plasma lens and the PWFA, and a trailing witness beam that is accelerated in the PWFA and focused by the plasma lens. The witness beam must be matched into the PWFA to achieve emittance preservation, so it is primarily the witness beam's properties that we consider here. While a complete picture of the drive beam dynamics are beyond the scope of this paper, this model will also describe the dynamics of the bulk of the drive beam within the fully depleted ion column.

In the highly nonlinear blowout regime, the entirety of the witness beam and the bulk of the drive beam are focused by an ion cavity that is fully depleted of electrons. The evolution of an electron beam in an ion cavity has already been thoroughly described in the literature $[6-9,29]$. In order for the plasma lens to be considered "thin", the lens must be significantly shorter than one betatron period along the axial dimension, and the plasma density must be rapidly truncated on its upstream and downstream ends. The plasma is then treated as an axisymmetric focusing lens following the standard formalism, where the transverse electron dynamics in the plasma lens can be represented by Hill's equation:

$$
x^{\prime \prime}+K(z) x=0
$$

Here, $x$ represents the transverse displacement from the center of the ion cavity, $z$ is the beam propagation axis, and $K$ is the focusing strength of the plasma given by $K(z)=k_{\beta}^{2}$. The focusing is purely linear in $x$ due to the spatial uniformity of the ion density in the blowout cavity. 
TABLE I. Comparison between focusing elements for a $10 \mathrm{GeV}$ electron beam with $\Delta \psi=0.1$.

\begin{tabular}{lccc}
\hline \hline Focusing element & $K\left[\mathrm{~m}^{-2}\right]$ & $L[\mathrm{~mm}]$ & $f[\mathrm{~cm}]$ \\
\hline Conventional Quadrupole & 0.3 & 180 & 1000 \\
Permanent Magnetic Quadrupole & 150 & 8.2 & 81 \\
Passive Thin Plasma Lens & 88400 & 0.34 & 3.3 \\
\hline \hline
\end{tabular}

The focal length of the thin plasma lens is given by

$$
f \equiv \frac{1}{K L}=\frac{1}{2 \pi r_{e}} \frac{\gamma_{b}}{n_{p} L}
$$

as previously noted in Refs. [26,30], where it is assumed that the betatron phase advance in the lens is small: $\Delta \psi \equiv \sqrt{K} L \ll 1$. For a nonuniform longitudinal plasma density profile, the effective focal length can be calculated by following the standard compound lens formalism, integrating over the density profile:

$$
f=\frac{1}{2 \pi r_{e}} \frac{\gamma_{b}}{\int n_{p}(z) d z} .
$$

Here we can consider how the focal length compares between passive thin plasma lenses, conventional quadrupole magnets, and PMQs. We assume an equivalent phase advance through each, $\Delta \psi=0.1$, so that all three optics are in the thin regime. The smallest focal length $f=$ $(\sqrt{K} \Delta \psi)^{-1}$ is then dependent on maximizing the focusing strength $K$. Table I summarizes the differences in focusing strength $K$, lens length $L$, and focal length $f$ for a $10 \mathrm{GeV}$ electron beam focused by a conventional quadrupole magnet with field gradient $G=1 \mathrm{~T} / \mathrm{m}$, a PMQ with $G=500 \mathrm{~T} / \mathrm{m}$, and a passive thin plasma lens with density $n_{p}=10^{17} \mathrm{~cm}^{-3}$. It can be seen that the laser-ionized, beam-driven, passive thin plasma lens can focus a relativistic electron beam with orders of magnitude greater strength than either of the magnetic focusing devices.

The beta function at the waist of the electron beam after the thin plasma lens, $\beta_{L}^{*}$, can be expressed in terms of the beam's Courant-Snyder parameters, $\beta_{0}, \alpha_{0}$, and $\gamma_{0}$, at the entrance to the plasma lens:

$$
\beta_{L}^{*}=\frac{1}{K^{2} L^{2} \beta_{0}+2 K L \alpha_{0}+\gamma_{0}},
$$

and the waist location after the lens is

$$
z_{w}=\frac{K L \beta_{0}+\alpha_{0}-L \gamma_{0}}{K^{2} L^{2} \beta_{0}+2 K L \alpha_{0}+\gamma_{0}},
$$

These equations represent the thin lens approximation of the thick lens expressions from transfer matrix formalism:

$$
\tilde{\beta}_{L, \text { thick }}^{*}=\frac{1}{\tilde{\beta}_{0} \sin ^{2} \tilde{L}+\tilde{\gamma}_{0} \cos ^{2} \tilde{L}+\alpha_{0} \sin (2 \tilde{L})},
$$

and

$$
\tilde{z}_{w, \text { thick }}=\frac{\left(\tilde{\beta}_{0}-\tilde{\gamma}_{0}\right) \sin \tilde{L} \cos \tilde{L}+\alpha_{0} \cos (2 \tilde{L})}{\tilde{\beta}_{0} \sin ^{2} \tilde{L}+\tilde{\gamma}_{0} \cos ^{2} \tilde{L}+\alpha_{0} \sin (2 \tilde{L})},
$$

where the tildes indicate dimensionless parameters normalized by a factor of $\sqrt{K}$ or $1 / \sqrt{K}$ as appropriate. The respective equations for thin lenses are found by setting $\tilde{L} \ll 1$.

The task of the plasma lens is to reduce the beam's final beta function with respect to the natural vacuum waist size that would be achieved in the absence of the plasma lens, $\beta_{v}^{*}$. It is useful to quantify the beta function magnification factor $\mathcal{M}$ in terms of $\beta_{v}^{*}$ and the incoming beta function at the start of the plasma lens, $\beta_{0}$ :

$\mathcal{M}=\frac{\beta_{L}^{*}}{\beta_{v}^{*}}=\frac{1}{K^{2} L^{2} \beta_{0} \beta_{v}^{*} \pm 2 K L \sqrt{\beta_{0} \beta_{v}^{*}}\left(1-\frac{\beta_{v}^{*}}{\beta_{0}}\right)^{1 / 2}+1}$,

where the sign in the denominator is the sign of $\alpha_{0}$. Noting that $\beta_{0}>\beta_{v}^{*}$, and assuming that $\beta_{v}^{*}$ is fixed, it can be seen that maximum demagnification occurs when $\beta_{0}$ is large, which is true for any thin, linear focusing element.

There are a few constraints on the plasma lens with respect to the blowout regime. First, the plasma must be sufficiently underdense relative to the drive beam to allow the production of a fully nonlinear blowout wake. Second, the period of the wake must be long enough to contain the witness bunch in the first blowout cavity. In practice, the second requirement will restrict the upper limit of plasma density to the nominal density of the target PWFA plasma stage. Lower densities will also function properly since the witness beam will be well within the first blowout cavity. Third, the blowout wake must be wide enough to contain the beam. The third constraint requires $k_{p} \sigma_{r}<1$, where $\sigma_{r}$ is the rms transverse spot size of the beam [31]. This can be expressed in terms of the dimensionless parameter $\chi$ :

$$
\chi \equiv K \beta_{0}^{2}<\frac{\beta_{0}}{2 \varepsilon_{n}},
$$

where $\varepsilon_{n}$ is the normalized transverse emittance of the beam, here considered to be symmetric in $x$ and $y$. If $\beta_{0}=\beta_{v}^{*}$, the magnification then becomes

$$
\mathcal{M}=\frac{1}{(\Delta \psi)^{2} \chi+1} .
$$

It can be seen from Eq. (10) that the magnification of the thin plasma lens is ultimately limited by the ratio $\beta_{0} / \varepsilon_{n}$ through $\chi$.

Chromatic aberrations will appear for any beam with finite energy spread, which presents the final limitation to the demagnification of the beam spot size. To quantify this effect, we utilize the chromatic amplitude given in Ref. [32]: 


$$
W^{2}=\left(\frac{\partial \alpha}{\partial \delta}-\frac{\alpha}{\beta} \frac{\partial \beta}{\partial \delta}\right)^{2}+\left(\frac{1}{\beta} \frac{\partial \beta}{\partial \delta}\right)^{2}
$$

The ratio of the projected geometric emittance at the exit of the lens, $\varepsilon_{f}$, to the initial geometric emittance, $\varepsilon_{0}$, is then given by

$$
\frac{\varepsilon_{f}}{\varepsilon_{0}} \approx \sqrt{1+W^{2} \sigma_{E}^{2}}
$$

where $\partial / \partial \delta$ are derivatives with respect to a relative energy offsets $\delta$ centered at $\delta=0$ with a Gaussian rms spread $\sigma_{E}$.

Equation (11) is evaluated by letting $K \rightarrow K /(1+\delta)$. The chromatic amplitude reduces to a compact form which depends on the phase advance through the lens and the ratio of the incoming beta function to the initial beam emittance, captured in the parameter $\chi$ :

$$
W_{\text {thin }}^{2}=K^{2} L^{2} \beta_{0}^{2}=(\Delta \psi)^{2} \chi .
$$

The expression for geometric emittance growth in a passive thin lens from Eqs. (12) and (13) is identical to the definition of aberration power from Ref. [33] for a general rms focusing strength variation.

In addition to the geometric emittance growth within the plasma, the normalized emittance can increase rapidly for a tightly focused beam propagating in vacuum. This normalized emittance growth is quantified in Ref. [34] as

$$
\epsilon_{n}^{2}=\left\langle\gamma_{b}\right\rangle^{2}\left(s^{2} \sigma_{E}^{2} \sigma_{x^{\prime}}^{4}+\epsilon^{2}\right)
$$

where $s$ is the drift distance and $\sigma_{x^{\prime}}=\sqrt{\gamma \epsilon}$ is the beam's rms size in momentum space. The normalized emittance growth at the focus is written

$$
\epsilon_{n}^{2}=\left\langle\gamma_{L}\right\rangle^{2}\left(\left(K L \beta_{0}+\alpha_{0}-L \gamma_{0}\right)^{2} \sigma_{E}^{2}+1\right) \epsilon_{f}^{2}
$$

For a passive thin plasma lens of $f=3.3 \mathrm{~cm}$ focusing a beam of $\beta_{v}^{*}=\beta_{0}=5 \mathrm{~cm}$ and $\sigma_{E}=0.25 \%$, Eq. (12) predicts the geometric emittance to grow by a factor of $7 \times 10^{-6}$. Equation (15) also predicts the normalized emittance to grow further by a factor $7 \times 10^{-6}$. Under most realistic conditions, $\left(K L \beta_{0}+\alpha_{0}-L \gamma_{0}\right)^{2}$ will be on the order of 1 and $\sigma_{E}^{2}$ will be on the order of $10^{-4}$ or smaller, thus the chromatic emittance growth from the passive thin plasma lens will be negligibly small.

\section{PWFA BEAM MATCHING}

To demonstrate that a plasma lens can focus a beam for beam matching, we consider a PWFA with a $10 \mathrm{~cm}$-long uniform density flat-top region of $3 \times 10^{16} \mathrm{~cm}^{-3}$ and Gaussian density ramps on either side with a half-width at half-maximum of $\sigma_{h w}=2.54 \mathrm{~cm}$. For a $10 \mathrm{GeV}$ electron beam to be matched into this plasma source, it must have a vacuum waist beta function of $\beta^{*}=2.5 \mathrm{~cm}$ at a position $4.55 \mathrm{~cm}$ before the start of the flattop [16].

The smallest vacuum beta function that can be produced by the final focus quadrupole electromagnets at the upcoming FACET-II facility is $\beta_{v}^{*}=5 \mathrm{~cm}[35,36]$. An appropriate thin plasma lens can provide the additional focusing necessary to reach the matching beta function. By solving Eq. (10) with $\mathcal{M}=1 / 2$ and $n_{p}=3 \times 10^{16} \mathrm{~cm}^{-3}$, the required lens thickness is found to be $737 \mu \mathrm{m}$. The ideal location of the plasma lens can be inferred from Eq. (5), which gives the distance from the plasma lens to the new waist location.

Figure 1 shows the beta function evolution of a $10 \mathrm{GeV}$ electron beam as it propagates through the thin plasma lens and PWFA described above. The simulation treats the plasma as an axisymmetric, linear focusing element. The plasma axial density profile of lens is given in Sec. IV. Figure 1 also includes an identical plasma lens at the PWFA exit that acts to control the high-divergence outgoing beam. After the exit lens, the beam evolves as if propagating from a vacuum waist beta function of $\beta_{f}^{*} \simeq 5 \mathrm{~cm}$. Energy gain in the PWFA is modeled according to Ref. [16], and an increase of $1.72 \mathrm{GeV}$ for the witness beam slightly diminishes the focusing strength of the exit lens with respect to its counterpart at the PWFA entrance. This can be compensated for by simply adjusting the thickness or position of the exit lens to achieve the desired value of $\beta_{f}^{*}$.

Lastly, we investigate the experimental tolerance on plasma lens thickness and location. Figure 2 shows the ratio

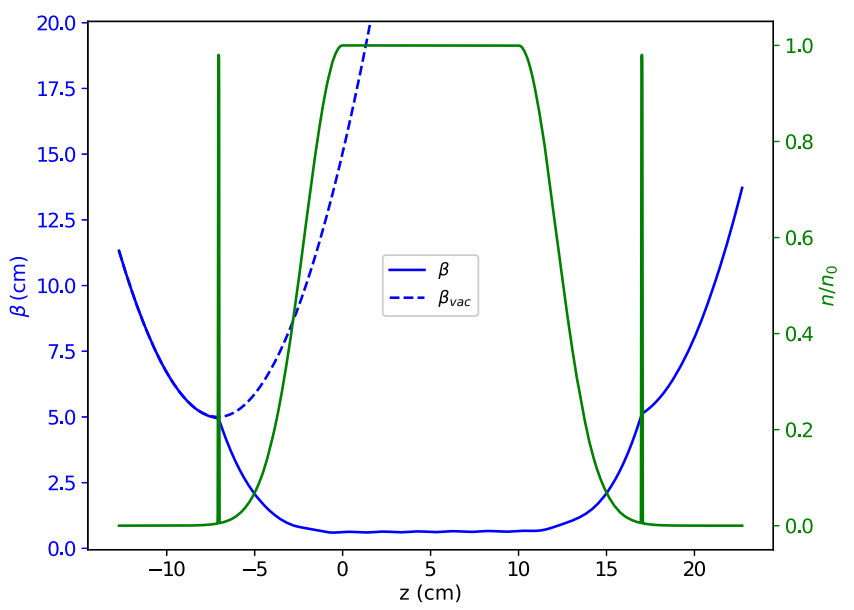

FIG. 1. Evolution of the beta function of a $10 \mathrm{GeV}$ electron beam through a plasma wakefield accelerator (PWFA) with density ramps of half-width $2.54 \mathrm{~cm}$ and peak density $n_{0}=3 \times 10^{16} \mathrm{~cm}^{-3}$. A thin plasma lens is located at the entrance and exit of the PWFA to match the incoming beam and mitigate its final divergence, respectively. The plasma density profile $n_{p}$ is given by the solid green line. The electron beam's beta function $\beta$ is given by the solid blue line. The beta function corresponding to vacuum propagation is given by the dashed blue line. 


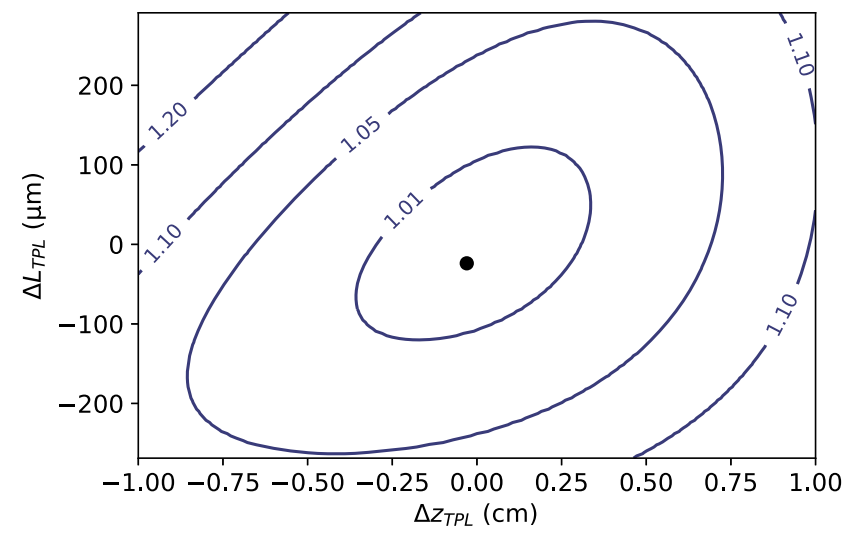

FIG. 2. Contour plot of the PWFA matching parameter $B_{m}$ over a range of errors in the plasma lens thickness $\Delta L_{\mathrm{TPL}}$ and plasma lens location $\Delta z_{\mathrm{TPL}}$. A tolerance of $1 \%$ saturated emittance growth, corresponding to the $B_{m}=1.01$ contour, permits an error of up to $\pm 100 \mu \mathrm{m}$ and $\pm 0.3 \mathrm{~cm}$ in the lens thickness and position, respectively. The filled circle represents the parameter set of the thin lens used in Fig. 1 with $B_{m}=1.0004$ and the axis origin represents the perfect matching value.

of the saturated emittance in the PWFA to the initial emittance $\left(B_{m}\right.$ [9]) for various combinations of error in the lens thickness $\left(\Delta L_{\mathrm{TPL}}\right)$ and lens location $\left(\Delta z_{\mathrm{TPL}}\right)$. In the FACET-II example considered here, the tolerance in both parameters is large. The lens thickness can vary by $\pm 100 \mu \mathrm{m}$ and the lens location can vary by $\pm 0.3 \mathrm{~cm}$ while still preserving the beam emittance to better than $1 \%$. These requirements are easily satisfied using the experimental setup described in Sec. IV.

An offset in Fig. 2 between the designed and optimal plasma lenses of $23.8 \mu \mathrm{m}$ in lens thickness and $0.3 \mathrm{~mm}$ in lens location is due to two effects.

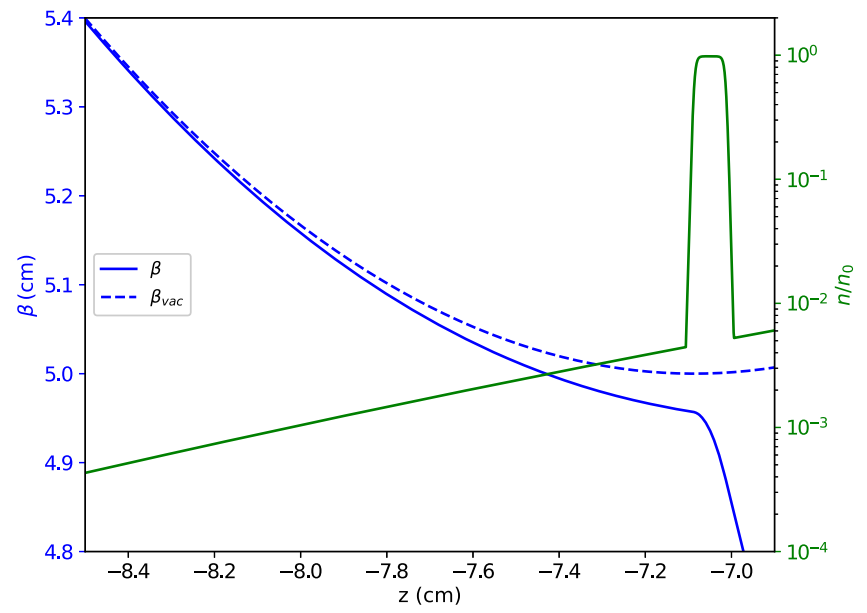

FIG. 3. Evolution of the electron beam beta function in the region of the plasma lens. Perturbative focusing from the low density plasma ramp shifts the beta function (solid blue) away from its theoretical vacuum trajectory (dashed blue). The plasma density profile $n_{p}$ is given in logarithmic scale by the solid green line, where $n_{0}=3 \times 10^{16} \mathrm{~cm}^{-3}$.
The designed lens thickness and position are shown in Fig. 2 with the filled circle, and the values is slightly different from the perfect matching condition (the axis origin) for two reasons. The first is because we used the thin lens approximation. If instead Eqs. (6) and (7) were used to design the lens, it would lead to a lens thickness $7.32 \mu \mathrm{m}$ greater than the design value.

The second effect originates from the perturbative focusing of the electron beam by the long tail of the PWFA plasma ramp prior to its arrival at the lens [9]. Figure 3 shows that the result of this effect is to create a beta function at the lens which is slightly smaller than the predicted vacuum value.

If necessary, both of the above effects can be analytically treated to generate a more accurate set of lens parameters for perfect beam matching, though as is demonstrated in Fig. 2, the simpler thin lens treatment should be more than sufficient for most purposes.

\section{THIN PLASMA LENS DESIGN}

To be effective for beam matching into a PWFA, a real plasma lens must meet the tolerance criteria described in the previous section. For this study, we show these demands can be met in the gas outflow of a typical jet nozzle. The open-source, computational fluid dynamics software, OPENFOAM [37], is used to simulate a

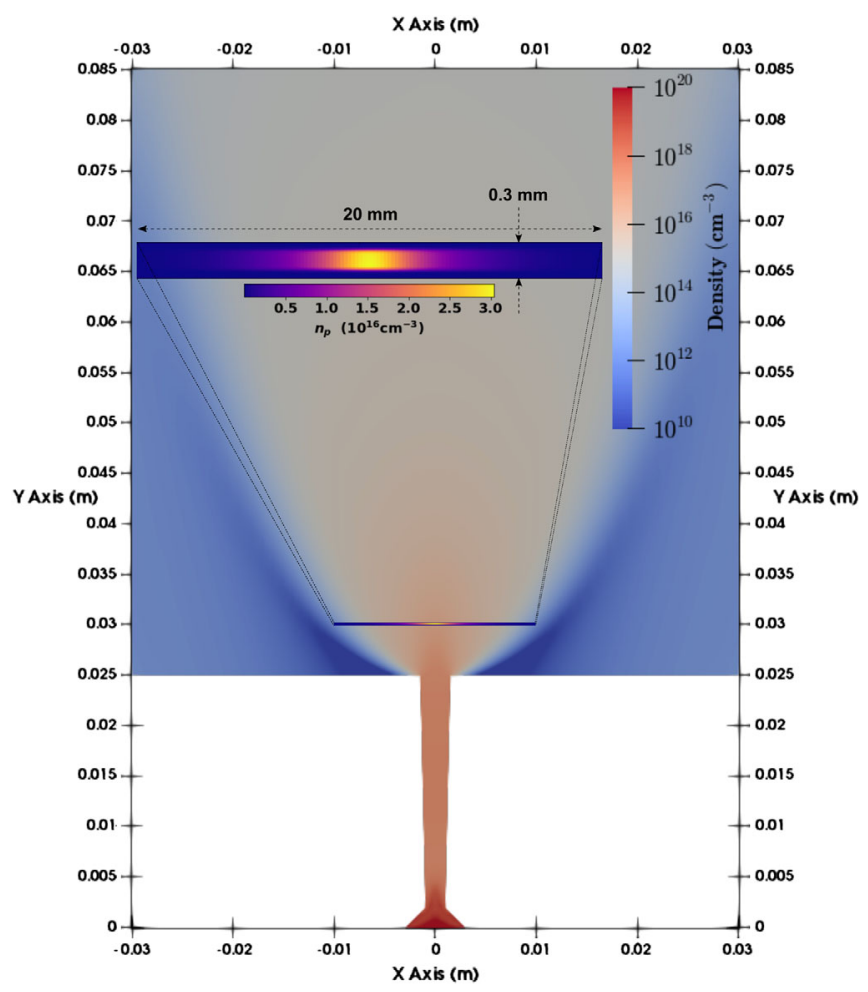

FIG. 4. Gas jet density profile at the mid-plane of the 3D OpenFOAM simulation at time step $140 \mu$ s. Insert: magnification of laser-ionized plasma density profile. An electron beam's trajectory would be into the page. 


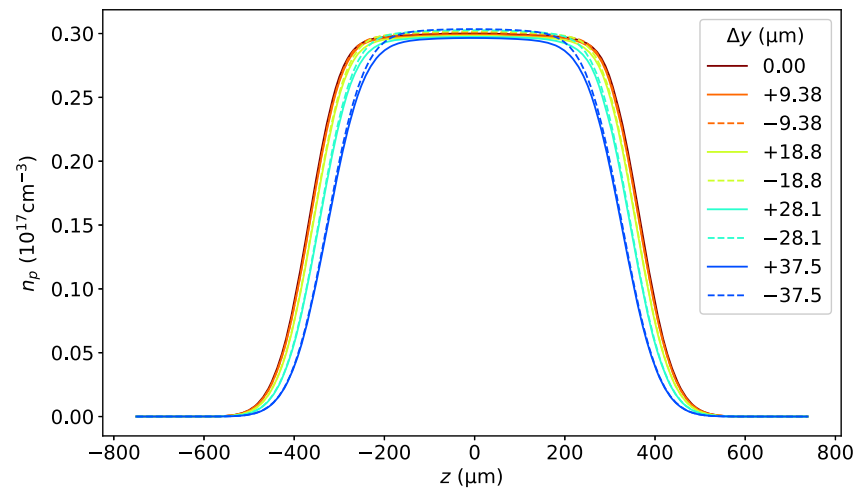

FIG. 5. Laser-ionized plasma density profile $\left(n_{p}\right)$ along the electron beam propagation axis at several vertical positions $(\Delta y)$ measured with respect to the center of the plasma lens.

compressible Argon gas propagating through a nozzle and out into a high vacuum space in $3 \mathrm{D}$. For gas jet simulation details and methodology see Appendix A. Figure 4 shows a cutaway final density profile along the gas jet midplane. The thin plasma lens will be formed in a volume located $5 \mathrm{~mm}$ above the nozzle, where the peak density is $3 \times 10^{16} \mathrm{~cm}^{-3}$. There, the horizontal density profile is approximately Lorentzian with $\gamma=4.02 \mathrm{~mm}$, and the vertical density profile varies linearly with a slope of $9.91 \times$ $10^{12} \mathrm{~cm}^{-3} / \mu \mathrm{m}$ over the region of interest.

The plasma is formed in the gas jet by laser ionization immediately prior to the arrival of the electron beam. To ionize a plasma lens with a suitable shape, we consider a Gaussian laser pulse of duration $35 \mathrm{fs}$, wavelength $800 \mathrm{~nm}$, and peak power $718 \mathrm{GW}$. This pulse propagates in the horizontal plane, perpendicular to the electron beam axis. It is focused by crossed cylindrical lenses, creating an asymmetric focus of spot sizes $125 \mu \mathrm{m}$ and $584 \mu \mathrm{m}$ in the horizontal and vertical plane, respectively. Ionization of the gas occurs via tunneling ionization and is calculated using ADK theory [38]. The resultant, singly-ionized plasma volume in Fig. 5 has a central length of $L=737 \mu \mathrm{m}$ along the electron beam propagation direction. Ionization defocusing effects of the plasma are considered in the simulation. To compensate for this effect and maintain the target plasma length, the laser power needs to be increased $1.3 \%$ to $727 \mathrm{GW}$.

The plasma lens is sufficiently wide in the dimensions transverse to the electron beam axis to contain the full blowout wake. The density is also sufficiently uniform over the transverse spatial extent of the electron beam (order $10 \mu \mathrm{m}$ radius) to provide axisymmetric, linear focusing. Figure 5 shows the longitudinal plasma density profile along the electron beam axis at different vertical positions measured with respect to the center of the plasma lens. It is convenient to describe the axial density profile in terms of the length of an equivalent step-function profile, which can be calculated using Eqs. (3) and (2). The effective plasma length thickness drops from $737 \mu \mathrm{m}$ in the center to $644 \mu \mathrm{m}$ and $659 \mu \mathrm{m}$ at locations $37.5 \mu \mathrm{m}$ above or below the center, respectively. The horizontal variation in effective thickness is much smaller than the vertical variation for this scenario. The effective thickness is reduced by $1.95 \%$ and $1.11 \%$ at the vertical positions of $\pm 14.1 \mu \mathrm{m}$ with respect to the center value. Meanwhile, the effective thickness is reduced by $0.04 \%$ and $1.14 \%$ at the horizontal positions of $\pm 156 \mu \mathrm{m}$ with respect to the center value. It will be shown in the next section that this degree of uniformity is enough to produce aberrationfree focusing for the considered electron beam.

\section{PIC SIMULATIONS}

The performance of the passive thin plasma lens is quantified precisely with a 3D particle in cell (PIC) simulation using the code VsIM [39]. The laser-ionized plasma profile calculated in the previous section is used in the PIC simulation along with a two-bunch (drive and witness) electron beam modeled on the projected parameters (Table II) for FACET-II. Details of the PIC simulation are given in Appendix B.

Figure 6 shows the final transverse phase space distribution of the witness beam in the $x$ and $y$ planes at the waist following the plasma lens. The statistical rms size of the electron beam is $2.04 \mu \mathrm{m}$ and $2.05 \mu \mathrm{m}$ in the $x$ and $y$ dimensions, respectively. These values differ by less than $1 \%$ from the those of a Gaussian fit, $2.06 \mu \mathrm{m}$ and $2.07 \mu \mathrm{m}$, as shown in Fig. 6. The vacuum waist size without the lens is $2.86 \mu \mathrm{m}$ and $2.81 \mu \mathrm{m}$ in $x$ and $y$. The lens has therefore decreased the minimum spot size by a factor of $1 / \sqrt{2}$, corresponding to the intended halving of the beta function. The final waist location obtained from the simulations is $2.47 \mathrm{~cm}$ downstream of the lens, which agrees well with the thin lens theoretical calculation of $2.49 \mathrm{~cm}$. The final beam distribution in transverse phase space is symmetric and aberrationfree, as demonstrated by the perfectly Gaussian distributions shown in Fig. 6 . The witness beam's transverse emittance in the horizontal and vertical dimensions grew from $3.199 \mu \mathrm{mrad}$ to

TABLE II. Electron beam parameters used in PIC simulation based on projected parameters for FACET-II.

\begin{tabular}{lcc}
\hline \hline Property & Drive beam & Witness beam \\
\hline Charge & $1.5 \mathrm{nC}$ & $0.5 \mathrm{nC}$ \\
Energy & $10 \mathrm{GeV}$ & $10 \mathrm{GeV}$ \\
$\epsilon_{n x}$ & $3.4 \mu \mathrm{m} \mathrm{rad}$ & $3.2 \mu \mathrm{m} \mathrm{rad}$ \\
$\epsilon_{n y}$ & $3.0 \mu \mathrm{m} \mathrm{rad}$ & $3.1 \mu \mathrm{m} \mathrm{rad}$ \\
$\beta_{x, y}$ & $70 \mathrm{~cm}, 70 \mathrm{~cm}$ & $5 \mathrm{~cm}, 5 \mathrm{~cm}$ \\
$\alpha_{x, y}$ & $4.2,1.6$ & 0,0 \\
$\sigma_{z}$ & $5.2 \mu \mathrm{m}$ & $5.2 \mu \mathrm{m}$ \\
$\sigma_{\delta}$ & $0.1 \%$ & $0.1 \%$ \\
$\Delta z$ & & $150 \mu \mathrm{m}$ \\
\hline \hline
\end{tabular}




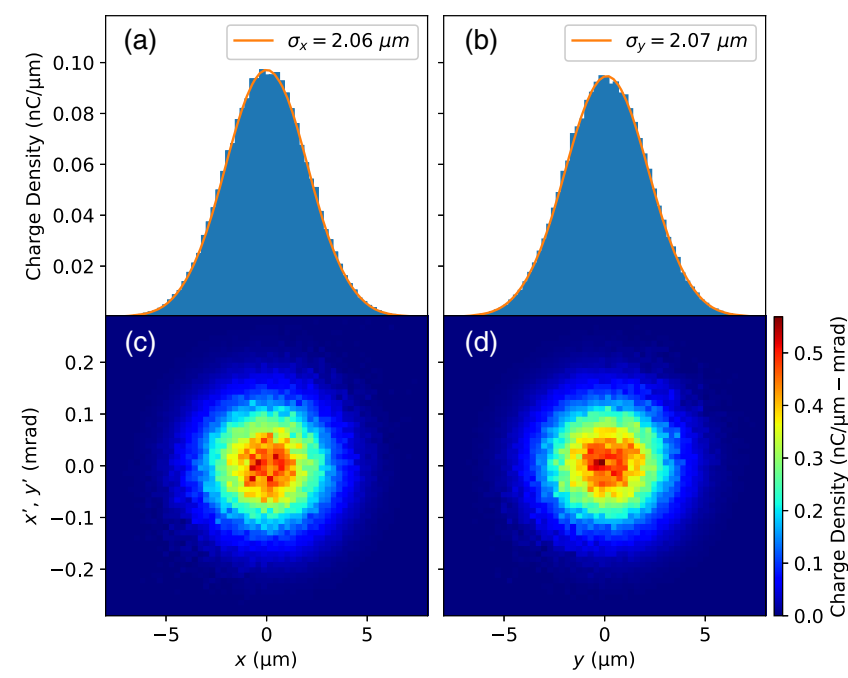

FIG. 6. Projected distribution of the horizontal (a) and vertical (b) coordinates of the electron witness beam at its waist following the passive thin plasma lens. A Gaussian fit to the profile is shown with the orange line. Transverse phase space in the horizontal (c) and vertical (d) planes exhibit perfectly Gaussian profiles in all transverse dimensions.

$3.201 \mu \mathrm{m} \mathrm{rad}$ and from $3.100 \mu \mathrm{m} \mathrm{rad}$ to $3.104 \mu \mathrm{m} \mathrm{rad}$, respectively. The change in the witness beam's energy and energy spread in the lens is negligibly small with centroid energy $\gamma_{b}=19570.5$ and rms energy spread $0.10010 \%$ before the plasma lens, and centroid energy $\gamma_{b}=19580.7$ and rms energy spread $0.10008 \%$ after the plasma lens.

\section{CONCLUSIONS}

The laser-ionized, beam-driven, underdense, passive thin plasma lens operating in the blowout regime can provide significant focusing of an electron beam beyond the capabilities of magnetic focusing devices. 3-D PIC simulations show good agreement with the analytic thin-lens model, and predict favorable performance for potential experimental implementation at FACET-II in the context of PWFA beam matching. The study shows that with the assistance of the thin plasma lens, the electron beam can reach a final beta function that is half that achievable by the FACET-II magnetic final focus system.

A simple method of implementation that utilizes crossed cylindrical lenses to focus a laser pulse into a gas jet is shown to be effective and robust against errors in the lens thickness and position. Other modes of laser ionization could also be implemented, such as axial propagation of an appropriately structured non-diffracting Bessel beam. The strength of the thin plasma lens is determined by the plasma density, the lens thickness, the beam rigidity, and the ratio of the electron beam's beta function to its normalized emittance at the entrance to the plasma lens.
Beam matching into a PWFA plasma source and divergence control at the exit of the plasma source are obvious applications for this thin plasma lens, although it could also be useful when operating with a single electron bunch to provide a smaller spot size at the final interaction point. Though some electrons at the head of the electron bunch would not experience the focusing force of the fully depleted ion cavity, the degree of head erosion could be mitigated by increasing the ratio of the incoming electron beam density to the plasma density. The single-bunch mode of operation could be a useful tool in studying high energy density science [40] and nonperturbative QED [41].

\section{ACKNOWLEDGMENTS}

This material is based upon work supported by the U.S. Department of Energy Office of Science (DOE-OOS), Office of High Energy Physics under Award No. DESC0017906. This research used resources of the National Energy Research Scientific Computing Center, a DOEOOS User Facility supported by the DOE-OOS under Contract No. DE-AC02-05CH11231.

\section{APPENDIX A: GAS JET SIMULATION DESCRIPTION}

The gas jet simulation was carried out using the code OPENFOAM [37]. The gas jet geometry is a conical nozzle with a $6 \mathrm{~mm}$ diameter gas inlet, $2 \mathrm{~mm}$ diameter throat, and $3 \mathrm{~mm}$ diameter nozzle exit. The distance from inlet to throat is $2 \mathrm{~mm}$ and the distance from throat to exit is $23 \mathrm{~mm}$. The outflow region is $60 \mathrm{~mm}$ from nozzle exit to the top and $60 \mathrm{~mm}$ wide radially. The lower boundary of this outflow region is a fixed wall.

At the inlet, the initial pressure is $41430 \mathrm{~Pa}$, or approximately $10^{19} \mathrm{~cm}^{-3}$. The inlet boundary condition is set to "totalPressure" which allows the pressure and gas velocity to evolve at the boundary. The background pressure is set to $0.04143 \mathrm{~Pa}$, or approximately $10^{13} \mathrm{~cm}^{-3}$, everywhere else. The outlet boundary conditions are "waveTransmissive" and are set to an infinite field of $0.04143 \mathrm{~Pa} 1 \mathrm{~m}$ beyond the boundary. Pressure fields have specific heats set to $\gamma=1.67$. Along the fixed walls, the pressure has a zero gradient boundary condition.

The temperature is initially set to $300 \mathrm{~K}$ everywhere, with a fixed value at the inlet and an "inletOutlet" boundary condition for the outlets and fixed walls. The velocity is initially set to zero everywhere, with a "pressureInletOutletVelocity" boundary at the inlet to match the pressure's inlet boundary condition. The outlets are set to an "inletOutlet" boundary condition, with a "noSlip" boundary condition along the fixed walls.

For computational efficiency, the simulation is run at a lower gas jet pressure than would be required by the example considered in this work. The results are then 
scaled by a factor of 7.411 to produce a gas density of $3 \times 10^{16} \mathrm{~cm}^{-3}$ at a location $5 \mathrm{~mm}$ above the nozzle exit.

\section{APPENDIX B: 3D PIC SIMULATION DESCRIPTION}

The 3D PIC simulation was carried out using the code VSIM [39]. The simulation used parameters for the drive and witness electron beams described in Table II with a moving window that is $300 \mu \mathrm{m}$ wide in the transverse dimension and $202 \mu \mathrm{m}$ long in the longitudinal dimension. The number of cells are 518 and 360 for the transverse and longitudinal dimensions, respectively. Particles are loaded at the front of the moving window, before the drive beam. The transverse boundaries of the window have a matched absorbing layer which is 8 cells deep. The simulation starts $700 \mu \mathrm{m}$ before the center of the plasma lens, and ends $600 \mu \mathrm{m}$ after the center of the lens. A timestep of $1.08 \times 10^{-15} \mathrm{~s}$ is calculated from the Courant condition.

The waist location of the witness beam is set to be at the center of the plasma lens. The plasma density profile in the longitudinal $z$ and vertical $y$ dimensions is loaded as a 2D fit from the laser-ionized plasma density profile in Sec. IV, with the density in $x$ assumed constant across the window dimensions. The 2D fit is a double-tanh profile that is elliptical in the $y-z$ plane and includes a slight linear gradient in $y$ due to the axial gas jet density profile:

$n(\vec{r})=n_{0}\left(\frac{1}{2}+\frac{1}{2} \tanh \frac{\zeta+a}{b}\right)\left(\frac{1}{2}-\frac{1}{2} \tanh \frac{\zeta-a}{b}\right)(d y+1)$

where

$$
\zeta(y, z)=\sqrt{(c y)^{2}+z^{2}},
$$

$n_{0}=2.9411 \times 10^{16} \mathrm{~cm}^{-3}, \quad a=376.41 \mu \mathrm{m}, \quad b=68.015 \mu \mathrm{m}$, $c=4.5828$, and $d=-4.5298 \times 10^{-4} \mu \mathrm{m}^{-1}$.

Particles within the beam are initially weighted from 0 to 1 in a Gaussian distribution. Once the witness beam has exited the plasma, we extract its phase space. The weighted electrons are then imported into our particle propagation code and the beam is evolved through vacuum to the waist position. This two-step procedure allows for VSIM to capture the complex dynamics of the plasma interaction, while saving significant computation time for modeling vacuum propagation.

[1] J. B. Rosenzweig, B. Breizman, T. Katsouleas, and J. J. Su, Acceleration and focusing of electrons in two-dimensional nonlinear plasma wake fields, Phys. Rev. A 44, R6189 (1991).

[2] M. J. Hogan, C. D. Barnes, C. E. Clayton, F. J. Decker, S. Deng, P. Emma, C. Huang, R. H. Iverson, D. K. Johnson,
C. Joshi, T. Katsouleas, P. Krejcik, W. Lu, K. A. Marsh, W. B. Mori, P. Muggli, C. L. O'Connell, E. Oz, R. H. Siemann, and D. Walz, Multi-GeV Energy Gain in a Plasma-Wakefield Accelerator, Phys. Rev. Lett. 95, 054802 (2005).

[3] I. Blumenfeld, C. E. Clayton, F.-J. Decker, M. J. Hogan, C. Huang, R. Ischebeck, R. Iverson, C. Joshi, T. Katsouleas, N. Kirby, W. Lu, K. A. Marsh, W. B. Mori, P. Muggli, E. Oz, R. H. Siemann, D. Walz, and M. Zhou, Energy doubling of $42 \mathrm{GeV}$ electrons in a metre-scale plasma wakefield accelerator, Nature (London) 445, 741 (2007).

[4] M. Litos et al., High-efficiency acceleration of an electron beam in a plasma wakefield accelerator, Nature (London) 515, 92 (2014).

[5] C. E. Clayton et al., Transverse Envelope Dynamics of a 28.5-GeV Electron Beam in a Long Plasma, Phys. Rev. Lett. 88, 154801 (2002).

[6] P. Chen, K. Oide, A. M. Sessler, and S. S. Yu, PlasmaBased Adiabatic Focuser, Phys. Rev. Lett. 64, 1231 (1990).

[7] P. Muggli, B. Blue, C. Clayton, S. Deng, F.-J. Decker, M. Hogan, C. Huang, R. Iverson, C. Joshi, T. Katsouleas, S. Lee, W. Lu, K. Marsh, W. Mori, C. O'Connell, P. Raimondi, R. Siemann, and D. Walz, Meter-Scale PlasmaWakefield Accelerator Driven by a Matched Electron Beam, Phys. Rev. Lett. 93, 014802 (2004).

[8] K. Floettmann, Adiabatic matching section for plasma accelerated beams, Phys. Rev. Accel. Beams 17, 054402 (2014).

[9] R. Ariniello, C. E. Doss, K. Hunt-Stone, J. R. Cary, and M. D. Litos, Transverse beam dynamics in a plasma density ramp, Phys. Rev. Accel. Beams 22 (2019).

[10] J. K. Lim, P. Frigola, G. Travish, J. B. Rosenzweig, S. G. Anderson, W. J. Brown, J. S. Jacob, C. L. Robbins, and A. M. Tremaine, Adjustable, short focal length permanentmagnet quadrupole based electron beam final focus system, Phys. Rev. Accel. Beams 8, 072401 (2005).

[11] F. Marteau, A. Ghaith, P. N'Gotta, C. Benabderrahmane, M. Vallau, C. Kitegi, A. Loulergue, J. Vtran, M. Sebdaoui, T. Andr, G. Le Bec, J. Chavanne, C. Vallerand, D. Oumbarek, O. Cosson, F. Forest, P. Jivkov, J. L. Lancelot, and M.E. Couprie, Variable high gradient permanent magnet quadrupole (QUAPEVA), Appl. Phys. Lett. 111, 253503 (2017).

[12] N. Barov and J. B. Rosenzweig, Propagation of short electron pulses in underdense plasmas, Phys. Rev. E 49, 4407 (1994).

[13] K. Marsh, C. Clayton, D. Johnson, C. Huang, C. Joshi, W. Lu, W. Mori, M. Zhou, C. Barnes, F.-J. Decker, M. Hogan, R. Iverson, P. Krejcik, C. O'Connell, R. Siemann, D. Walz, S. Deng, T. Katsouleas, P. Muggli, and E. Oz, Beam matching to a plasma wake field accelerator using a ramped density profile at the plasma boundary, in Proceedings of the 2005 Particle Accelerator Conference (IEEE, New York, 2005), pp. 2702-2704, https://doi.org/10.1109/ PAC.2005.1591234.

[14] T. Mehrling, J. Grebenyuk, F. S. Tsung, K. Floettmann, and J. Osterhoff, Transverse emittance growth in staged laserwakefield acceleration, Phys. Rev. Accel. Beams 15, 111303 (2012). 
[15] I. Dornmair, K. Floettmann, and A. R. Maier, Emittance conservation by tailored focusing profiles in a plasma accelerator, Phys. Rev. Accel. Beams 18, 041302 (2015).

[16] M. D. Litos, R. Ariniello, C. E. Doss, K. Hunt-Stone, and J. R. Cary, Beam emittance preservation using Gaussian density ramps in a beam-driven plasma wakefield accelerator, Phil. Trans. R. Soc. A 377, 2151 (2019).

[17] X. L. Xu, J. F. Hua, Y. P. Wu, C. J. Zhang, F. Li, Y. Wan, C.-H. Pai, W. Lu, W. An, P. Yu, M. J. Hogan, C. Joshi, and W. B. Mori, Physics of Phase Space Matching for Staging Plasma and Traditional Accelerator Components Using Longitudinally Tailored Plasma Profiles, Phys. Rev. Lett. 116, 124801 (2016).

[18] P. Chen, A possible final focusing mechanism for linear colliders, Part. Accel. 20, 171 (1987).

[19] G. Hairapetian, P. Davis, C. E. Clayton, C. Joshi, S. C. Hartman, C. Pellegrini, and T. Katsouleas, Experimental Demonstration of Dynamic Focusing of a Relativistic Electron Bunch by an Overdense Plasma Lens, Phys. Rev. Lett. 72, 2403 (1994).

[20] H. Nakanishi, Y. Yoshida, T. Ueda, T. Kozawa, H. Shibata, K. Nakajima, T. Kurihara, N. Yugami, Y. Nishida, T. Kobayashi, A. Enomoto, T. Oogoe, H. Kobayashi, B. S. Newberger, S. Tagawa, K. Miya, and A. Ogata, Direct Observation of Plasma-Lens Effect, Phys. Rev. Lett. 66, 1870 (1991).

[21] A. Marocchino et al., Experimental characterization of the effects induced by passive plasma lens on high brightness electron bunches, Appl. Phys. Lett. 111, 184101 (2017).

[22] J. van Tilborg, S. Steinke, C. Geddes, N. Matlis, B. Shaw, A. Gonsalves, J. Huijts, K. Nakamura, J. Daniels, C. Schroeder, C. Benedetti, E. Esarey, S. Bulanov, N. Bobrova, P. Sasorov, and W. Leemans, Active Plasma Lensing for Relativistic Laser-Plasma-Accelerated Electron Beams, Phys. Rev. Lett. 115, 184802 (2015).

[23] R. Pompili et al., Experimental characterization of active plasma lensing for electron beams, Appl. Phys. Lett. 110, 104101 (2017).

[24] C. A. Lindstrøm, E. Adli, G. Boyle, R. Corsini, A. E. Dyson, W. Farabolini, S. M. Hooker, M. Meisel, J. Osterhoff, J.-H. Röckemann, L. Schaper, and K. N. Sjobak, Emittance Preservation in an Aberration-Free Active Plasma Lens, Phys. Rev. Lett. 121, 194801 (2018).

[25] C. A. Lindstrøm and E. Adli, Analytic plasma wakefield limits for active plasma lenses, arXiv:1802.02750.

[26] M. C. Thompson, H. Badakov, J. B. Rosenzweig, G. Travish, N. Barov, P. Piot, R. Fliller, G. M. Kazakevich, J. Santucci, J. Li, and R. Tikhoplav, Observations of lowaberration plasma lens focusing of relativistic electron beams at the underdense threshold, Phys. Plasmas 17, 073105 (2010).

[27] R. Lehe, C. Thaury, E. Guillaume, A. Lifschitz, and V. Malka, Laser-plasma lens for laser-wakefield accelerators, Phys. Rev. Accel. Beams 17, 121301 (2014).
[28] C. Thaury, E. Guillaume, A. Dpp, R. Lehe, A. Lifschitz, K. Ta Phuoc, J. Gautier, J.-P. Goddet, A. Tafzi, A. Flacco, F. Tissandier, S. Sebban, A. Rousse, and V. Malka, Demonstration of relativistic electron beam focusing by a laserplasma lens, Nat. Commun. 6, 6860 (2015).

[29] J. B. Rosenzweig, P. Schoessow, B. Cole, C. Ho, W. Gai, R. Konecny, S. Mtingwa, J. Norem, M. Rosing, and J. Simpson, Demonstration of electron beam self-focusing in plasma wake fields, Phys. Fluids B 2, 1376 (1990).

[30] J. J. Su, T. Katsouleas, J. M. Dawson, and R. Fedele, Plasma lenses for focusing particle beams, Phys. Rev. A 41, 3321 (1990).

[31] R. Govil, W. P. Leemans, E. Y. Backhaus, and J. S. Wurtele, Observation of Return Current Effects in a Passive Plasma Lens, Phys. Rev. Lett. 83, 3202 (1999).

[32] C. A. Lindstrøm and E. Adli, Design of general apochromatic drift-quadrupole beam lines, Phys. Rev. Accel. Beams 19, 071002 (2016).

[33] J. B. Rosenzweig and P. Chen, Beam optics of a selffocusing plasma lens, Phys. Rev. D 39, 2039 (1989).

[34] M. Migliorati, A. Bacci, C. Benedetti, E. Chiadroni, M. Ferrario, A. Mostacci, L. Palumbo, A. R. Rossi, L. Serafini, and P. Antici, Intrinsic normalized emittance growth in laser-driven electron accelerators, Phys. Rev. Accel. Beams 16, 011302 (2013).

[35] C. Joshi, E. Adli, W. An, C. E. Clayton, S. Corde, S. Gessner, M. J. Hogan, M. Litos, W. Lu, K. A. Marsh, W. B. Mori, N. Vafaei-Najafabadi, B. Oshea, X. Xu, G. White, and V. Yakimenko, Plasma wakefield acceleration experiments at FACET II, Plasma Phys. Controlled Fusion 60, 034001 (2018).

[36] Technical Design Report for the FACET-II Project at SLAC National Accelerator Laboratory, SLAC National Accelerator Laboratory Technical Design Report No. SLAC-R1072, 2016, p. 1340171, https://doi.org/10.2172/1340171.

[37] H. G. Weller, G. Tabor, H. Jasak, and C. Fureby, A tensorial approach to computational continuum mechanics using object-oriented techniques, Comput. Phys. 12, 620 (1998).

[38] M. V. Ammosov, N. B. Delone, and V. P. Krainov, Tunnel ionization of complex atoms and of atomic ions in an alternating electromagnetic field, Sov. Phys. JETP 64, 4 (1986).

[39] C. Nieter and J. R. Cary, VORPAL: A versatile plasma simulation code, J. Comput. Phys. 196, 448 (2004).

[40] C. Joshi, B. Blue, C. E. Clayton, E. Dodd, C. Huang, K. A. Marsh, W. B. Mori, S. Wang, M. J. Hogan, C. OConnell, R. Siemann, D. Watz, P. Muggli, T. Katsouleas, and S. Lee, High energy density plasma science with an ultrarelativistic electron beam, Phys. Plasmas 9, 1845 (2002).

[41] V. Yakimenko, S. Meuren, F. Del Gaudio, C. Baumann, A. Fedotov, F. Fiuza, T. Grismayer, M. J. Hogan, A. Pukhov, L. O. Silva, and G. White, On the Prospect of Studying Nonperturbative QED with Beam-Beam Collisions, Phys. Rev. Lett. 122, 190404 (2019). 